\title{
A KINETIC FORMULATION \\ OF MULTIDIMENSIONAL SCALAR CONSERVATION LAWS AND RELATED EQUATIONS
}

\author{
P. L. LIONS, B. PERTHAME, AND E. TADMOR
}

\section{SUMMARY}

1. Introduction

2. Kinetic formulation of entropy solutions

3. Applications to compactness and regularity of entropy solutions

4. Moments estimates and bounds on entropy solutions

5. Related equations

Appendix: Velocity-Averaging revisited

\section{INTRODUCTION}

We consider a multidimensional scalar conservation law

$$
\frac{\partial \rho}{\partial t}+\sum_{i=1}^{N} \frac{\partial}{\partial x_{i}}\left(A_{i}(\rho)\right)=0 \text { for } t \geq 0, x \in \mathbb{R}^{N}
$$

where $A_{1}, \ldots, A_{N}$ are $C^{2, \alpha}$ functions from $\mathbb{R}$ into $\mathbb{R}$ (for some $\alpha>0$ ), and we shall restrict our attention to the entropy solutions of (1), that is, say, bounded solutions of (1) -in the sense of distributions-which satisfy entropy inequalities as introduced by Lax [22]. In the case of (1), the entropy inequalities take the form

$$
\frac{\partial S(\rho)}{\partial t}+\sum_{i=1}^{N} \frac{\partial}{\partial x_{i}}\left(\eta_{i}(\rho)\right) \leq 0 \quad \text { in } \mathscr{D}^{\prime}
$$

for any convex function $S$, where $\eta_{i}$ is defined by

$$
\eta_{i}(t)=\int_{0}^{t} S^{\prime}(\sigma) A_{i}^{\prime}(\sigma) d \sigma \quad \forall t \in \mathbb{R} .
$$

As shown by Kružkov [21] (see also Smoller [31] for a presentation of this classical theory together with more details on the origin and mathematical analysis

Received by the editors October 2, 1992.

1991 Mathematics Subject Classification. Primary 35L65, 35L67, 35Q20, 76P05.

Key words and phrases. Scalar multidimensional conservation laws, entropy solutions, kinetic formulation, pseudo-maxwellian, velocity averaging, compactness, oscillations, genuine nonlinearity, regularity results, degenerate nonlinear parabolic equations.

The third author was partially supported by ONR Contract No. N00014-91-J-1076. 
of (1) and further references), there exists a unique solution of (1) satisfying (2) and initial conditions

$$
\left.\rho\right|_{t=0}=\rho^{0}(x) \text { in } \mathbb{R}^{N}
$$

where $\rho^{0}$, for instance, belongs to $L^{\infty} \cap L^{1}\left(\mathbb{R}^{N}\right)$ and solutions are seeked in $L^{\infty}\left(\mathbb{R}^{N} \times(0, \infty)\right) \cap C\left([0, \infty) ; L^{1}\left(\mathbb{R}^{N}\right)\right)$ for example.

In this work, we introduce a new formulation of (1), (2). We call this formulation a kinetic one by its analogy with the classical kinetic models such as Boltzmann or Vlasov models (see, for instance, Cercignani [7] or the survey by DiPerna and Lions [12]). In particular, we add a real-valued variable called velocity, denoted by $v$, and the unknown becomes a "density-like" function $f(x, v, t)$. Then, we introduce the following equation (and restrictions) on $f$ :

$$
\frac{\partial f}{\partial t}+a(v) \cdot \nabla_{x} f=\frac{\partial m}{\partial v} \quad \text { in } \mathscr{D}^{\prime}\left(\mathbb{R}^{N} \times \mathbb{R} \times(0, \infty)\right)
$$

with

$$
f=\chi_{\rho(x, t)}(v) \text { for some function } \rho(x, t)
$$

and

$$
m \text { is a nonnegative bounded measure on } \mathbb{R}_{x}^{N} \times \mathbb{R}_{v} \times(0, \infty) \text {. }
$$

Here and everywhere below, the velocity distributions (or profiles) $\chi_{\alpha}(v)$ are parametrized by $\alpha \in \mathbb{R}$ and are defined by

$$
\chi_{\alpha}(v)= \begin{cases}1 & \text { if } 0<v \leq \alpha \\ -1 & \text { if } \alpha \leq v<0 \\ 0 & \text { otherwise }\end{cases}
$$

and we denote by $a$ the vector-valued function given by $a(v)=\left(a_{1}(v), \ldots\right.$, $\left.a_{N}(v)\right)$ and $a_{i}(v)=A_{i}^{\prime}(v) \quad(1 \leq i \leq N)$. Finally, $x \cdot y$ denotes the scalar product between $x$ and $y$ in $\mathbb{R}^{N}$. Again, by analogy with the classical kinetic theory of gases, $\chi_{\alpha}(v)$ can be called a pseudo-maxwellian. Then, the equality (6) can be looked at as a (nonlinear) constraint on $f$, which creates a Lagrange multiplier term in the linear equation (5), namely, $\partial m / \partial v$ with $m$ satisfying (7).

Let us finally observe that (6), together with the definition (8) of $\chi_{\alpha}(v)$, immediately yields

$$
\rho(x, t)=\int_{\mathbb{R}} f(x, v, t) d v \quad \text { on } \mathbb{R}^{N} \times(0, \infty) .
$$

Before commenting further on this kinetic model, let us mention how this paper is organized. In $\S 2$, we show that (5)-(7) defines a well-posed problem with an initial condition

$$
\left.f\right|_{t=0}=\chi_{\rho^{0}(x)}(v) \quad \text { in } \mathbb{R}_{x}^{N} \times \mathbb{R}_{v}
$$

which is equivalent to (1), (2), (4).

In $\S \S 3$ and 4 we will present various applications of this new formulation of scalar conservation laws consisting of compactness, estimates, and regularity 
results for solutions of (1), (2). These results are completely new in our multidimensional setting (i.e., when $N \geq 2$ ) and even in dimension $N=1$, some of our regularity results appear to be new too. In particular, we will introduce nondegeneracy conditions on vector-field $\left(A_{i}\right)$ which correspond to a multidimensional extension of genuine nonlinearity type conditions (or a related condition introduced by Tartar [33]). Under such a condition, we will show that bounded sequences of solutions of (1), (2) are in fact compact in $L_{\mathrm{loc}}^{1}$, extending thus a celebrated result due to Tartar [32] obtained in [32] by the study of oscillations (via Young's measures) and the use of compensated-compactness theory (see Tartar [33], Murat [25]).

Finally, in $\S 5$, we briefly explain how our analysis can be extended to related equations like scalar conservation laws with source terms or second-order degenerate parabolic equations in conservation form, i.e.,

$$
\frac{\partial \rho}{\partial t}+\sum_{i=1}^{N} \frac{\partial}{\partial x_{i}}\left(A_{i}(\rho)\right)-\sum_{i, j=1}^{N} \frac{\partial^{2}}{\partial x_{i} \partial x_{j}}\left(A_{i j}(\rho)\right)=0 \text { in } \mathbb{R}^{N} \times(0, \infty)
$$

where $\left(A_{i j}\right) \in C^{1,1}$ satisfies

$$
\left(A_{i j}^{\prime}(t)\right) \geq 0 \text { for all } t \in \mathbb{R} .
$$

At this stage, it is worth indicating the main tool that allows us to extract new information on compactness and regularity from the kinetic formulation of $(1),(2)$. Our analysis will rely upon the so-called velocity averaging lemmata that allow to obtain "more" regularity on macroscopic quantities like $\rho(x, t)$ which are velocity averages or averages of $f$ in $v$. The first results in that direction were discovered by Golse, Perthame, and Sentis [19] and Golse, Lions, Perthame, and Sentis [18], various extensions are to be found in DiPerna and Lions [12], Gérard [15, 16]; and a rather complete theory was developed by DiPerna, Meyer, and Lions [13]. However, it is, necessary for our purposes to adapt a bit of the results and methods of [13] to our setting, adaptation that we present in an Appendix.

We would like to conclude this long introduction by a few semihistorical remarks and comments that might shed some light on the somewhat unusual approach to "macroscopic equations" proposed here. First of all, one can say that the links between Continuum Mechanics models (or macroscopic equations), such as heat, Navier-Stokes, or Euler equations, and kinetic models arising at a more detailed description of the evolution of "matter" (a gas for instance) have been emphasized since the very early stages of kinetic theory. This link consisting in a limit (hydrodynamical limit, mean free path going to 0 ) has been investigated by the very founders (Maxwell, Boltzmann, Hilbert, etc.) of the kinetic theory of gases. And it was always hoped that this limit would reveal and illuminate some of the complex phenomena present at the macroscopic level. Illustrations of this constant hope can be found in various works in $\mathrm{Nu}$ merical Analysis or Scientific Computations like the study and development of Boltzmann schemes for Fluid Mechanics models. Our work can thus be seen as a small contribution to that general program. Let us also point out that not only can we analyse a hydrodynamical limit but we also can write down 
at the macroscopic level a kinetic equation involving a "density-like" function whose velocity distribution is the "equilibrium" density (here $\chi_{\alpha}(v)$ ) obtained by minimization of "entropy-like" functionals.

Let us also remark that extensions of our work to more general conservation laws (systems) are not clear and that, up to now, our approach seems to rely very much on some "scalar features". Next, we would like to point out that our work can be seen as a sequel of the work by Perthame and Tadmor [29] investigating the hydrodynamical limit of BGK type models (5)-(7) and their relation with (1), (2). Also, this paper introduces a setting which, in some sense, is a continuous version of some time-derivations of (1), (2) studied by Brenier [5] or Giga and Miyakawa [17]. Finally, the usefulness of velocity averaging lemmata for hydrodynamical limits has already been demonstrated by various works that include Bardos, Golse, Perthame, and Sentis [2] for radiative transfer models, Bardos, Golse, and Levermore [1] for Boltzmann equation, and Golse and Poupaud [20] for semiconductor models.

Let us finally mention that most of the results proven here were announced in the Comptes-Rendus de l'Académie des Sciences de Paris (Sér. I 312 (1991), 9-102). Extensions to $2 \times 2$ systems have been obtained recently by the authors [37].

\section{KINETIC FORMULATIONS OF ENTROPY SOLUTIONS}

We begin with a result showing the relations between entropy solutions of (1), (2) and the kinetic system (5)-(7).

Theorem 1. (1) Let $\rho \in L^{\infty}\left(\mathbb{R}_{x}^{N} \times(0, \infty)\right) \cap C\left(0, \infty ; L^{1}\left(\mathbb{R}_{x}^{N}\right)\right)$ solve (1), $(2)$, and set $f(x, v, t)=\chi_{\rho(x, t)}(v)$. Then, $f \in C\left(0, \infty ; L^{1}\left(\mathbb{R}_{x}^{N} \times \mathbb{R}_{v}\right)\right) \cap$ $L^{\infty}\left(\mathbb{R}_{x}^{N} \times(0, \infty) ; L^{1}\left(\mathbb{R}_{v}\right)\right)$ solves (5) for some $m$ satisfying (7) supported in $\mathbb{R}_{x}^{N} \times\left[-R_{0}, R_{0}\right] \times(0, \infty)$ where $R_{0}=\|\rho\|_{L^{\infty}}$. In addition, if we denote by $\mu$ the bounded nonnegative measure on $\mathbb{R}$ defined by

$$
\int_{\mathbb{R}} g(v) d \mu(v)=\int_{\mathbb{R}_{x}^{N} \times \mathbb{R}_{v} \times(0, \infty)} g(v) d m \text { for all } g \in \mathscr{D}(\mathbb{R})
$$

then $\mu \in L^{1}(\mathbb{R}) \cap L^{\infty}(\mathbb{R})$ and we have

$$
\left\{\begin{array}{l}
\int_{\mathbb{R}} \mu d v \leq \frac{1}{2} \sup _{t \geq 0}\|\rho(t)\|_{L^{2}\left(\mathbb{R}_{x}^{N}\right)}^{2} \quad \text { a.e. in } \mathbb{R} . \\
\mu \leq \sup _{t>0}\|\rho(t)\|_{L^{1}\left(\mathbb{R}_{x}^{N}\right)}
\end{array}\right.
$$

(2) Let $f \in C\left(0, \infty ; L^{1}\left(\mathbb{R}_{x}^{N} \times \mathbb{R}_{v}\right)\right) \cap L^{\infty}\left(\mathbb{R}_{x}^{N} \times(0, \infty)\right.$ and $L^{1}\left(\mathbb{R}_{v}\right)$ solve (5), (6) for some $m$ satisfying (7), and set $\rho(x, t)=\int_{\mathbb{R}} f(x, v, t) d v$. Then $\rho \in L^{\infty}\left(\mathbb{R}_{x}^{N} \times(0, \infty)\right) \cap C\left(0, \infty ; L^{1}\left(\mathbb{R}_{x}^{N}\right)\right)$ solves $(1)$, (2) .

Remarks. (1) Of course, $\rho \in C\left([0, \infty) ; L^{1}\left(\mathbb{R}_{x}^{N}\right)\right)$ if and only if

$$
f \in C\left([0, \infty) ; L^{1}\left(\mathbb{R}_{x}^{N} \times \mathbb{R}_{v}\right)\right),
$$

in which case the suprema in (13) may be replaced by their values at $t=0$. 
(2) The above result still holds if we only assume $\rho \in L^{\infty}\left(\mathbb{R}_{x}^{N} \times(0, \infty)\right)$, in which case $m$ is a nonnegative measure on $\mathbb{R}_{x}^{N} \times \mathbb{R}_{v} \times(0, \infty)$ satisfying the following bounds for all $R \in(0, \infty)$ :

$$
\sup _{z \in \mathbb{R}^{N}} \int 1_{(0, R)}(t) 1_{z+B_{R}}(x) d m(x, v, t)<\infty .
$$

Before we begin the proof of Theorem 1, let us mention the following immediate consequence of uniqueness results for (1), (2) due to Kružkov [21].

Corollary 1. Let $\rho^{0} \in L^{\infty} \cap L^{1}\left(\mathbb{R}^{N}\right)$. Then there exists a unique

$$
f \in C\left([0, \infty) ; L^{1}\left(\mathbb{R}_{x}^{N} \times \mathbb{R}_{v}\right)\right) \cap L^{\infty}\left(\mathbb{R}_{x}^{N} \times(0, \infty) ; L^{1}\left(\mathbb{R}_{v}\right)\right)
$$

solving (5), (6), (9) with $m$ satisfying (7).

Remarks. (1) The measure $m$, in the above result, is also uniquely determined since $\partial m / \partial v$ is by (5), and then the uniqueness of $m$ follows from (7).

(2) The measure $m$ can be interpreted as the Kružkov's entropy dissipation. Indeed, choosing for $S$ the Kružkov entropy $(\rho-k)_{+}$, we find that the righthand side of (2) is exactly (formally) $-m(x, k, t)$ since the kinetic formulation gives the value $\left\langle S^{\prime}(v), \partial_{v} m\right\rangle=-m(x, k, t)$. The equality

$$
m(x, v, t)=-\left\{\frac{\partial}{\partial t}(\rho-v)^{+}+\sum_{i=1}^{n} \frac{\partial}{\partial x_{i}}\left[\operatorname{sign}^{+}(\rho-v)\left(A_{i}(\rho)-A_{i}(v)\right)\right]\right\}
$$

(in the sense of distributions) can easily be justified.

(3) Let us observe that $\int_{\mathbb{R}}\left(\chi_{\alpha}(v)-\chi_{\beta}(v)\right)^{+} d v=(\alpha-\beta)^{+}$so that we have for all $t \geq 0$

$$
\begin{aligned}
\int_{\mathbb{R}^{N}}\left(\rho_{1}-\rho_{2}\right)^{+} d x & =\int_{\mathbb{R}^{N+1}}\left(f_{1}-f_{2}\right)^{+} d x d v \\
& \leq \int_{\mathbb{R}^{N}}\left(\rho_{1}^{0}-\rho_{2}^{0}\right)^{+} d x=\int_{\mathbb{R}^{N+1}}\left(f_{1}^{0}-f_{2}^{0}\right)^{+} d x d v
\end{aligned}
$$

where $\left(\rho_{1}, f_{1}\right)$ and $\left(\rho_{2}, f_{2}\right)$ are the solutions corresponding to initial conditions $\left(\rho_{1}^{0}, f_{1}^{0}\right)$ and $\left(\rho_{2}^{0}, f_{2}^{0}\right)$ respectively (recall that $f_{i}^{0}=\chi_{\rho_{i}^{0}}(v)$ for $\left.i=1,2\right)$. This fact again is deduced from Theorem 1 and classical comparison-uniqueness results for (1), (2).

We now turn to the proof of Theorem 1 . We begin with the proof of the second assertion, which is based upon the following remark

$$
\int \chi_{\alpha}(v) \varphi(v) d v=\int_{0}^{\alpha} \varphi(\sigma) d \sigma \quad \text { for all } \varphi \in C(\mathbb{R}), \alpha \in \mathbb{R}
$$

Then, at least formally, (1), (2) follow from multiplying (5) by $1, \varphi^{\prime}(v)$ where $\varphi$ is a $C^{2}$ convex function, and integrating over $v \in \mathbb{R}$. The sign in (2) is then deduced from the sign of $m$ (7) and the convexity of $\varphi$. Now, to justify this formal computation, the only difficulty consists in justifying the integration by parts over $\mathbb{R}$ in the "product" $\varphi(v) \partial m / \partial v$. In order to do so, we first remark that without loss of generality we may assume that $\varphi(0)=0$ and $\varphi^{\prime \prime}$ 
has bounded support so that $\varphi^{\prime}$ is bounded. Then, let $\psi \in C_{0}^{\infty}(\mathbb{R})$ satisfy $0 \leq \psi \leq 1, \psi \equiv 1$ on $[-1,+1]$, Supp $\psi \subset[-2,+2]$, and set, for $n \geq 1$, $\psi_{n}(v)=\psi(v / n)$. Next, let $g \in \mathscr{D}\left(\mathbb{R}_{x}^{N} \times(0, \infty)\right), g \geq 0$. We want to show

$$
-\int \varphi(\rho) \frac{\partial g}{\partial t}+\sum_{i=1}^{N} \eta_{i}(\rho) \frac{\partial g}{\partial x_{i}} d x d t \leq 0 .
$$

This inequality will yield (2) while (1) is shown by similar computations.

Then, we multiply (5) (in distributions sense) by $g(x, t) \varphi^{\prime}(v) \psi_{n}(v)$ and find

$$
-\int \varphi^{n}(\rho) \frac{\partial g}{\partial t}+\sum_{i=1}^{N} \eta_{i}^{n}(\rho) \frac{\partial g}{\partial x_{i}} d x d t=-\int g\left(\varphi^{\prime \prime} \psi_{n}+\varphi^{\prime} \psi_{n}^{\prime}\right) d m
$$

where $\varphi^{n}(t)=\int_{0}^{t} \varphi^{\prime}(v) \psi_{n}(\sigma) d \sigma, \eta_{i}^{n}(t)=\int_{0}^{t} a_{i}(\sigma) \varphi^{\prime}(v) \psi_{n}(v) d v$.

Next, we observe that $(7)$ implies

$$
\int g \varphi^{\prime \prime} \psi_{n} d m \geq 0, \quad\left|\int g \varphi^{\prime} \psi_{n}^{\prime} d m\right| \leq \frac{C}{n}
$$

for some $C \geq 0$ independent of $n$. Then, (17) yields (16) since $\varphi^{n}(\rho), \eta_{i}^{n}(\rho)$ converge respectively to $\varphi(\rho), \eta_{i}(\rho)$ in $L_{\mathrm{loc}}^{1}$.

We now turn to the proof of the first assertion in Theorem 1 . We denote by $T$ the distribution of order at most 1 defined by $\partial f / \partial t+a(v) \cdot \nabla_{x} f$. Observe that $\operatorname{Supp} T \subset \mathbb{R}_{x}^{N} \times\left[-R_{0}, R_{0}\right] \times(0, \infty)$. In addition, since we have by (15)

$$
\int_{\mathbb{R}} f d v=\rho, \quad \int_{\mathbb{R}} a_{i}(v) f d v=A_{i}(\rho)
$$

we deduce from (1) that $T$ satisfies

$$
\langle T, g \otimes 1\rangle=0 \text { for all } g(x, t) \in \mathscr{D}\left(\mathbb{R}_{x}^{N} \times(0, \infty)\right) \text {. }
$$

In view of the restriction on the support of $T$ mentioned above, we may define a distribution $m$ on $\mathbb{R}_{x}^{N} \times \mathbb{R}_{v} \times(0, \infty)$ by

$$
\langle m, g\rangle=-\left\langle T, \int_{-\infty}^{v} g(x, w, t) d w\right\rangle \quad \forall g \in \mathscr{D}\left(\mathbb{R}_{x}^{N} \times \mathbb{R}_{v} \times(0, \infty)\right) .
$$

Clearly, $m$ is of order at most 1 and, because of (18), is supported in $\mathbb{R}_{x}^{N} \times$ $\left[-R_{0}, R_{0}\right] \times(0, \infty)$. Of course, $T=\partial m / \partial v$ in $\mathscr{D}^{\prime}$.

Therefore, if $g(x, t) \in \mathscr{D}\left(\mathbb{R}_{x}^{N} \times(0, \infty)\right) \geq 0$ and $\psi \in \mathscr{D}\left(\mathbb{R}_{v}\right) \geq 0$, we may set $\varphi(v)=\int_{0}^{v}\left(\int_{-\infty}^{t} \psi(\sigma) d \sigma\right) d t$ and deduce from (19)

$$
\begin{aligned}
\langle m, g \otimes \psi\rangle & =-\left\langle T, g \otimes \varphi^{\prime}\right\rangle \\
& =\left\langle f, \frac{\partial g}{\partial t} \otimes \varphi^{\prime}+\sum_{i=1}^{N} \frac{\partial g}{\partial x_{i}} \otimes\left(a_{i} \varphi^{\prime}\right)\right\rangle \\
& =\int f(x, v, t)\left[\varphi^{\prime}(v) \frac{\partial g}{\partial t}+\sum_{i=1}^{N} a_{i}(v) \varphi^{\prime}(v) \frac{\partial g}{\partial x_{i}}\right] d x d v d t \\
& =\int \varphi(\rho) \frac{\partial g}{\partial t}+\sum_{i=1}^{N} \eta_{i}(\rho) \frac{\partial g}{\partial x_{i}} d x d t \geq 0
\end{aligned}
$$


in view of (15) and (2). This sign, by an easy adaptation of the proof of Schwarz lemma on nonnegative distributions, is enough to ensure that $m$ is a nonnegative measure on $\mathbb{R}_{x}^{N} \times \mathbb{R}_{v} \times(0, \infty)$ (supported in $\mathbb{R}_{x}^{N} \times\left[-R_{0}, R_{0}\right] \times$ $(0, \infty))$. The above computation shows in particular in view of $(18)$ that

$$
\langle m, g \otimes 1\rangle=\int \frac{\rho^{2}}{2} \frac{\partial g}{\partial t}+\sum_{i=1}^{N} \bar{\eta}_{i}(\rho) \frac{\partial g}{\partial x_{i}} d x d t,
$$

where $\bar{\eta}_{i}(t)=\int_{0}^{t} a_{i}(\sigma) \sigma d \sigma$.

Next, we wish to justify the choice of $g(x, t)=g(t)$ in (20). This is done by considering $g_{n}(x, t)=\bar{g}(x / n) g(t)$ with $\bar{g} \in \mathscr{D}\left(\mathbb{R}^{N}\right), 0 \leq \bar{g} \leq 1, \bar{g} \equiv 1$ if $|x| \leq 1, g \in \mathscr{D}(0, \infty), g \geq 0$, from which we deduce

$$
0 \leq \int_{\mathbb{R}_{x}^{N} \times \mathbb{R}_{v} \times(0, \infty)} g(t) d m(x, v, t)=\int \frac{\rho^{2}}{2} g^{\prime}(t) d x d t .
$$

We then deduce easily from (21) the fact that $m$ satisfies (7) and the first bound in (13) by observing that $\int \rho^{2} d x$ is a nonincreasing function of $t>0$ in view of $(2)$.

There only remains to show that $\mu \in L^{\infty}(\mathbb{R})$ and satisfies the second bound in (13). This is shown by an argument quite similar to that above replacing $g \otimes 1$ by $g \otimes \psi_{\varepsilon}(w)$ where $w$ is arbitrary in $\mathbb{R}$ and $\psi_{\varepsilon}=\frac{1}{\varepsilon} \psi(\dot{\bar{\varepsilon}}), \psi \in C_{0}^{\infty}(\mathbb{R})$, $\psi \geq 0, \int_{\mathbb{R}} \psi d v=1$. We then obtain

$$
0 \leq \mu * \psi_{\varepsilon} \leq \sup _{0<t} \int_{\mathbb{R}^{N}} \varphi_{\varepsilon}(\rho(t)) d x
$$

where $\varphi_{\varepsilon}(t)=\int_{0}^{t} \int_{0}^{s} \psi_{\varepsilon}(w-\sigma) d \sigma d s$ for all $t \in \mathbb{R}$. We then conclude easily since $0 \leq \varphi_{\varepsilon}(t) \leq|t|$ on $\mathbb{R}$ for all $\varepsilon>0$.

We now conclude this section by recalling a Boltzmann-like (or more precisely a BGK-like) model proposed by Perthame and Tadmor [29] which yields (1), (2) as an "hydrodynamical limit" and in fact "converges to (5)-(7)". It consists in solving

$$
\begin{gathered}
\frac{\partial f^{\varepsilon}}{\partial t}+a(v) \cdot \nabla_{x} f^{\varepsilon}=\frac{1}{\varepsilon}\left(\chi_{\rho^{\varepsilon}}(v)-f^{\varepsilon}\right)=0 \quad \text { in } \mathbb{R}_{x}^{N} \times \mathbb{R}_{v} \times(0, \infty), \\
\rho^{\varepsilon}(x, t)=\int_{\mathbb{R}} f^{\varepsilon}(x, v, t) d v \quad \text { in } \mathbb{R}_{x}^{N} \times(0, \infty)
\end{gathered}
$$

together with the initial condition

$$
\left.f^{\varepsilon}\right|_{t=0}=\chi_{\rho_{0}(x)}(v) \text { in } \mathbb{R}_{x}^{N} \times \mathbb{R}_{v} .
$$

By following Kružkov's theory [21] of conservation laws (1), (2), it was shown in [29] that, if $\rho_{0} \in L^{1}\left(\mathbb{R}^{N}\right) \cap L^{\infty}\left(\mathbb{R}^{N}\right)$, then $\rho^{\varepsilon}$ converges in $L^{1}\left(\mathbb{R}^{N} \times(0, T)\right)$ $(\forall T<\infty)$ to the solution $\rho$ of $(1),(2),(4)$. Notice that $(23),(24)$ is a semilinear, nonlocal, hyperbolic (first-order) equation which is rather simple to solve for $\varepsilon>0$ fixed. Let us remark finally that $f^{\varepsilon}$ satisfies the following properties

$$
\left|f^{\varepsilon}\right| \leq 1 \quad \text { a.e., } \quad \operatorname{Supp} f^{\varepsilon} \subset \mathbb{R}_{x}^{N} \times\left[-R_{0}, R_{0}\right] \times[0, \infty),
$$




$$
f^{\varepsilon} \geq 0 \quad \text { a.e. for } v \geq 0, \quad f^{\varepsilon} \leq 0 \quad \text { a.e. for } v \leq 0 .
$$

We then want to show

Theorem 2. The solution $f^{\varepsilon}$ of (23)-(25) converges in $L^{1}\left(\mathbb{R}_{x}^{N} \times \mathbb{R}_{v} \times(0, T)\right)$ $(\forall T<\infty)$ to the solution of (5)-(7), (9). In addition, $\frac{1}{\varepsilon}\left(\chi_{\rho^{\varepsilon}}-f^{\varepsilon}\right)$ may be written as $\partial m_{\varepsilon} / \partial v$ where $m_{\varepsilon}$ is a nonnegative measure on $\mathbb{R}_{x}^{N} \times \mathbb{R}_{v} \times[0, \infty)$ bounded independently of $\varepsilon$ which converges weakly to the measure $m$ uniquely determined by (5)-(7).

Remarks. (1) In fact, one can show that $f^{\varepsilon}$ converges in $C\left([0, T] ; L^{1}\right) \quad(\forall T<$ $\infty)$ to $f$ and that $m^{\varepsilon}$ is a tight family of bounded nonnegative measures.

(2) It would be useful to have more qualitative information on $m$, for instance, its support. For example, one may deduce from the above result and from [29] that $m$ vanishes on open sets of the form $\{(x, v, t) /(x, t) \in \mathscr{O}$, $v \in \mathbb{R}\}$ where $\mathscr{O}$ is an open set on which $\rho$ is locally Lipschitz. In other words, $m$ is "supported by the shocks". Another way to check this fact is to use the equality mentioned in Remark (2) following Corollary 1.

Proof of Theorem 2. In view of the results recalled above, we only need to show that $\frac{1}{\varepsilon}\left(\chi_{\rho^{\varepsilon}}-f^{\varepsilon}\right)$ can be written as $\partial m_{\varepsilon} / \partial v$ where $m_{\varepsilon}$ is a nonnegative measure bounded independently of $\varepsilon>0$. This fact can be shown in several ways. One way is to observe that if $\alpha \in \mathbb{R}, g(v) \in L^{1}(\mathbb{R})$ satisfies

$$
\begin{cases}-1 \leq g \leq 0 & \text { for } v \leq 0 \\ 0 \leq g \leq 1 & \text { for } v \geq 0 \\ \int_{\mathbb{R}} g(v) d v=\alpha & \end{cases}
$$

then

$$
\left\{\begin{array}{l}
\chi_{\alpha}(v)-g(v)=q^{\prime}(v) \\
\text { for some nonnegative, bounded, continuous function } q .
\end{array}\right.
$$

Indeed, set $q(v)=\int_{-\infty}^{v}\left(\chi_{\alpha}(w)-g(w)\right) d w$. In the case when $\alpha>0$, the other case being treated similarly, we see that $q$ is nondecreasing on $(-\infty, \alpha)$ and nonincreasing on $(\alpha,+\infty)$. And we conclude since $q(-\infty)=0$ and $q(+\infty)=$ $\alpha-\int_{\mathbb{R}} g d v=0$.

Another possible proof consists in recalling the following elementary lemma (due to Y. Brenier [5]) and copying the proof of Theorem 1 (which then becomes simpler since distributions theory is no more needed).

Lemma 1. Let $\alpha \in \mathbb{R}$ and let $\varphi$ be a $C^{1}$ convex function on $\mathbb{R}$ such that $\varphi^{\prime}$ is bounded. Then, $\chi_{\alpha}(v)$ is a minimizer of (30)

$$
\inf \left\{\int_{\mathbb{R}} \varphi^{\prime}(v) g(v) d v / g \in L^{1}(\mathbb{R}), \int_{\mathbb{R}} g d v=\alpha, 0 \leq g \operatorname{sign} v \leq 1 \text { a.e. on } \mathbb{R}\right\} \text {. }
$$

In addition, $\chi_{\alpha}(v)$ is the unique minimizer of (30) if $\varphi^{\prime}$ is strictly increasing on $\mathbb{R}$. 
Remark. In fact, Lemma 1 is equivalent to (29) since, if we denote by $\mu$ the bounded nonnegative measure given by $\varphi^{\prime \prime}$, we have easily

$$
\int_{\mathbb{R}} \varphi^{\prime}(v)\left[g(v)-\chi_{\alpha}(v)\right] d v=\int_{\mathbb{R}} q(v) d \mu(v) \geq 0 .
$$

And the uniqueness of $\chi_{\alpha}$ follows since, arguing by contradiction, if $g$ is another minimizer, we find that $q$ vanishes on the closure of $\operatorname{Supp}(\mu)$, which is $\mathbb{R}$ since $\varphi^{\prime}$ is strictly increasing on $\mathbb{R}$.

Of course, (29) or Lemma 1 is probably the key point making possible the kinetic formulation (5)-(7) of scalar conservation laws (1), (2).

\section{APPLICATIONS TO COMPACTNESS AND REGULARITY OF ENTROPY SOLUTIONS}

In this section we deduce from the kinetic formulation (5)-(7) new informations on compactness and regularity properties of entropy solutions of (1), (2). We begin with compactness properties and consider a family $\rho^{\varepsilon} \in$ $C\left(0, \infty ; L^{1}\left(\mathbb{R}^{N}\right)\right) \cap L^{\infty}\left(\mathbb{R}^{N} \times(0, \infty)\right)$ of solutions of $(1),(2)$. We assume that $\rho^{\varepsilon}$ is bounded on $\mathbb{R}^{N} \times(0, \infty)$ and in $L^{1}\left(\mathbb{R}^{N}\right)$ uniformly in $t$, independently of $\varepsilon$ and denote by $R_{0}$ a common bound in $L^{\infty}$. $\mathbb{R}^{N}$

We next introduce a nondegeneracy assumption on the curve $(v \mapsto a(v))$ in

$$
\begin{aligned}
& \operatorname{meas}\left\{|v|<R_{0}, \tau+a(v) \cdot \xi=0\right\}=0 \\
& \qquad \text { for all }(\tau, \xi) \in \mathbb{R} \times \mathbb{R}^{N} \text { such that } \tau^{2}+\xi^{2}=1 .
\end{aligned}
$$

The assumption means that the curve $(v \mapsto a(v))$ cannot "stay" in a given hyperplane. When $N=1$, this condition is closely related (it is in fact stronger) to a condition introduced by Tartar [32], namely, that $a$ is not constant on a nonempty open interval. In higher dimensions, the strict analogue of Tartar's condition (and its implications!) was not known and a related attempt can be found in Engquist [14]. With this condition, we can prove

Theorem 3. Under assumption (31), $\rho^{\varepsilon}$ is relatively compact in $L^{1}\left((0, T) \times B_{R}\right)$ $(\forall T, R<\infty)$.

Remarks. (1) In view of Theorem 1, Theorem 3 is shown if we prove that

$$
\int_{\mathbb{R}} f^{\varepsilon} \psi d v \text { is relatively compact in } L_{\text {loc }}^{1}\left(\mathbb{R}_{x}^{N} \times(0, \infty)\right)
$$

where

$$
f^{\varepsilon}=\chi_{\rho^{\varepsilon}(x, t)}(v), \quad \psi=1_{\left[-R_{0}, R_{0}\right]}(v) .
$$

(2) We will give examples of fluxes $A$ satisfying condition (31) later on. At this stage, let us only mention that, when $N=1,(31)$ reduces to

$$
\operatorname{meas}\left\{|v|<R_{0}, a(v)=\lambda\right\}=0 \text { for all } \lambda \in \mathbb{R} \text {. }
$$

(3) It is worth remarking that one can use this compactness result at the level of the BGK model considered in the preceding section to deduce directly the existence of solutions, providing thus an alternate route to existence theory (which is in some sense independent of $\mathrm{BV}$ estimates or $L^{1}$-contraction arguments). 
(4) It is obvious that the following choice of $A$ provides an example where (31) does not hold. We choose $A(v)=\left(v^{2}, v^{2}\right)$ in two dimensions $(n=2)$ so that $(1)$ reads

$$
\frac{\partial \rho}{\partial r}+\frac{\partial}{\partial x}\left(\rho^{2}\right)+\frac{\partial}{\partial y}\left(\rho^{2}\right)=0
$$

And since all functions $\rho_{0}(x-y)$ are steady solutions of that equation, it is obvious that Theorem 3 cannot hold (as remarked in [14]). This example shows that a condition like (31) is necessary.

We are going to show below that Theorem 3 is a straightforward consequence of the following general compactness result, which is a variant of the results shown in DiPerna, Lions, and Meyer [13] (the proof of this result is sketched in the appendix).

Theorem B. Let $1<p \leq 2$, let $f$ be bounded in $L_{\text {loc }}^{p}\left(\mathbb{R}_{x}^{N} \times \mathbb{R}_{v} \times(0, \infty)\right)$, let $g$ belong to a compact set of $L^{p}\left(\mathbb{R}_{x}^{N} \times \mathbb{R}_{v} \times(0, \infty)\right)$, and let $r \geq 0$. We assume that $f$ satisfies

$$
\frac{\partial f}{\partial t}+\sum_{i=1}^{N} a_{i}(v) \frac{\partial f}{\partial x_{i}}=\left(-\Delta_{x, t}+1\right)^{1 / 2}\left(-\Delta_{v}+1\right)^{r / 2} g \text { in } \mathscr{D}^{\prime}
$$

where $a \in C_{\mathrm{loc}}^{l, \alpha}$ with $l=r, \alpha=1$ if $r$ is an integer, $l=[r]$, and $\alpha=r-l$ if $r$ is not an integer. Let $\psi \in L^{p^{\prime}}\left(\mathbb{R}_{v}\right)$ with compact support.

Finally, we assume that a satisfies

$$
\begin{aligned}
\operatorname{meas}\{v \in \operatorname{Supp} \psi, \tau+a(v) \cdot \xi & =0\}=0 \\
\forall(\tau, \xi) & \in \mathbb{R} \times \mathbb{R}^{N} \text { with } \tau^{2}+|\xi|^{2}=1 .
\end{aligned}
$$

Then, $\int_{\mathbb{R}} f \psi d v$ belongs to a compact set of $L_{\mathrm{loc}}^{p}\left(\mathbb{R}_{x}^{N} \times(0, \infty)\right)$.

In order to deduce Theorem 3 from Theorem $B$, we use the kinetic formulation, i.e., we consider $f^{\varepsilon}=\chi_{\rho^{\varepsilon}}$ (see also the Remark (1) above) and only need to show that the associated Lagrange multipliers $\partial m_{\varepsilon} / \partial v$ can be written in the form of the right-hand side of (34). Recall also that we know from Theorem 1 that $m_{\varepsilon}$ is a bounded family of bounded nonnegative measures on $\mathbb{R}_{x}^{N} \times \mathbb{R}_{v} \times(0, \infty)$. Then, if we choose $p<(N+2) /(N+1)$, we know from Sobolev embeddings that $m_{\varepsilon}$ is bounded in $W^{-s, p}\left(\mathbb{R}_{x}^{N} \times \mathbb{R}_{v} \times(0, \infty)\right)$ for $1>s>(N+2) / p^{\prime}$. Notice that $s$ goes to $0_{+}$as $p$ goes to $1_{+}$. This easily yields (34) for $r>1+(N+2) / p^{\prime}$. And Theorem 3 then follows from Theorem B upon choosing $\psi(v)=1_{\left[-R_{0}, R_{0}\right]}(v)$.

Remark. We want to emphasize here some relationship between measure valued solutions, Young's measures and our kinetic formulation. This relationship can be seen by taking weak limits of entropy solutions $\rho^{\varepsilon}$ of (1), (2) as in Tartar [32] and considering (up to subsequences) the Young's measures $\nu_{x, t}$ associated to $\rho^{\varepsilon}$, namely, probability measures on $\mathbb{R}$, parametrized (in a measurable way) by $(x, t) \in \mathbb{R}^{N} \times(0, \infty)$, such that for all $\varphi \in C(\mathbb{R})$

$$
\varphi\left(\rho^{\varepsilon}\right) \underset{\varepsilon}{\rightarrow} \int \varphi(\lambda) d \nu_{x, t}(\lambda) \quad w-L^{\infty}\left(\mathbb{R}_{x}^{N} \times(0, \infty)\right)_{*} .
$$


Therefore, if $\left(f^{\varepsilon}, m^{\varepsilon}\right)$ converge weakly (in $L_{*}^{\infty}$, in the sense of measures) respectively to $(f, m)$, we see that $(5)$ or $(7)$ still hold. In addition, for any $\varphi \in C^{1}(\mathbb{R})$, we deduce from the above convergence

$$
\int \varphi(\lambda) d \nu_{x, t}(\lambda)-\varphi(0)=\int f \varphi^{\prime}(v) d v
$$

hence $\partial f / \partial v=\delta_{0}-\nu_{x, t}$ in $\mathscr{D}^{\prime}$ and $f=\int \chi_{\lambda}(v) d \nu_{x, t}(\lambda)$ a.e.

Then, (5)-(7) and these equalities give a formulation of the evolution of Young's measures which is in fact equivalent (by the same proof as in Theorem 1) to the notion of measure-valued solutions satisfying an entropy condition as defined for instance by DiPerna [11].

We now turn to regularity results for entropy solutions of (1), (2). We shall need to strengthen assumption (31). We consider an initial condition $\rho^{0} \in$ $L^{1} \cap L^{\infty}\left(\mathbb{R}^{N}\right)$ and let $R_{0}>\left\|\rho^{0}\right\|_{L^{\infty}\left(\mathbb{R}^{N}\right)}$. We shall use the following condition on $a$ :

$$
\begin{aligned}
& \exists \alpha \in(0,1], \exists C \geq 0, \quad \operatorname{meas}\left\{|v|<R_{0},|\tau+a(v) \cdot \xi| \leq \delta\right\} \leq C \delta^{\alpha} \\
& \quad \text { for } \delta \in(0,1),(\tau, \xi) \in \mathbb{R} \times \mathbb{R}^{N} \text { with } \tau^{2}+|\xi|^{2}=1
\end{aligned}
$$

Then, denoting by $\rho$ the entropy solution of (1), (2), (4) which belongs to $C\left([0, \infty) ; L^{1}\left(\mathbb{R}_{x}^{N}\right)\right) \cap L^{\infty}\left(\mathbb{R}_{x}^{N} \times(0, \infty)\right)$ and satisfies $\|\rho\|_{L_{x, t}^{\infty}}=\left\|\rho^{0}\right\|_{L^{\infty}\left(\mathbb{R}^{N}\right)}$, we have

Theorem 4. Under condition (36), we have for all $\varepsilon \in(0,1)$ and for all $s \in$ $(0, \alpha /(\alpha+2))$

$$
\begin{gathered}
\rho \in W_{\mathrm{loc}}^{s, p}\left(\mathbb{R}_{x}^{N} \times(0, \infty)\right) \\
\|\rho\|_{W^{s, p}\left(B_{1 / \varepsilon} \times(\varepsilon, 1 / \varepsilon)\right)} \leq C \quad \text { with } p=\frac{4+\alpha}{2+\alpha}, \\
\rho \in C\left((0, \infty) ; W_{\mathrm{loc}}^{s, 1}\left(\mathbb{R}^{N}\right)\right), \quad \text { and } \sup _{t \geq \varepsilon}\|\rho(t)\|_{W^{s, 1}\left(B_{1 / \varepsilon}\right)} \leq C
\end{gathered}
$$

for some $C \geq 0$ depending only on $\varepsilon, s, R_{0}$, and $\left\|\rho^{0}\right\|_{L^{1}\left(\mathbb{R}^{N}\right)}$.

Remarks (1). Such regularizing effects results appear to be completely new when $N \geq 2$. When $N=1$, various related regularizing effects have been obtained. First of all, when $A$ is strongly convex or concave, i.e., $A^{\prime \prime}>0$, then it is a classical fact that $\rho$ is in BV for $t>0$. Notice that in that case (36) holds with $\alpha=1$, and thus we do not recover fully this classical result. Other results of that sort have been shown when $N=1$-see, for example, Bénilan and Crandall [3], Liu and Pierre [24], Dafermos [9]. They all show that some nonlinear function of $\rho$ is in BV for $t>0$. Let us take a typical example taken from [3]: we consider the case when $A(t)=\alpha|t|^{m}$ if $t \geq 0,=\beta|t|^{m}$ if $t<0$ with $\alpha, \beta \in \mathbb{R}$, $m \geq 2$. Then, $A(\rho)$ is bounded in $\mathrm{BV}$ for positive time. But this in general does not imply any regularity on $\rho$. If, for instance, $\alpha=\beta \neq 0$, then a function $\rho$ taking only values +1 and -1 will be such that $A(\rho) \equiv 1$ ! Other regularity results can be found in Zumbrun [36] which for similar reasons are not strictly comparable to ours. This shows that such regularity results valid for 
very specific fluxes $A$ are not comparable to Theorem 4. Let us mention that, in the above example, by a direct examination based upon Lax [22]-Oleinik [26] formula, one can check that $u \in C\left(0, \infty ; W_{\mathrm{loc}}^{s, 1}\right)$ for all $s<1 /(m-1)$ (and this is sharp!). Let us also point out that (36) holds with $\alpha=1 /(m-1)$ if $m \geq 2$.

(2) We gave in the preceding remark various examples of fluxes $A$ satisfying (36). Let us mention a few other examples. Still if $N=1$, if $A(t)=\sin t$ or $A(t)=\cos t$, then (36) holds with $\alpha=\frac{1}{2}$. When $N \geq 2$, computing the sharp exponent $\alpha$ for which (36) holds is a delicate matter. Let us consider a few examples when $N=2$ : take first $a_{1}(v)=v, a_{2}(v)=e^{v}$ (i.e., $A_{1}=v^{2} / 2$, $A_{2}=e^{v}$, which are both strongly convex); then (36) holds with $\alpha=\frac{1}{2}$. If we take $a_{1}(v)=|v|^{m} v, a_{2}(v)=|v|^{p} v$ with $0<m \neq p$; then (36) holds with $\alpha=\max (1+m, 1+p)^{-1}$.

(3) It is clear that Theorem 4 is not sharp with respect to the "best" range of possible $s$. We conjecture that (38) holds if $s<\alpha$ (instead of $\alpha /(\alpha+2)$ ) while (37) holds for all $1 \leq p<\infty$ with $s<\alpha / p$ (instead of $\alpha /(\alpha+2)$ when $p=(4+\alpha) /(2+\alpha))$.

The proof of Theorem 4 that we postpone relies upon the following result, which is a variant of the results shown in DiPerna, Lions, and Meyer [13], variant whose proof is sketched in the appendix.

Theorem A. Let $1<p \leq 2$, let $1 \leq q \leq 2$, let a satisfy (36), and let $\psi \in$ $C_{0}^{\infty}(\mathbb{R})$ be supported in $\left(-R_{0}, R_{0}\right)$. Let $f \in W_{\text {loc }}^{\sigma, p}\left(\mathbb{R}_{x}^{N} \times \mathbb{R}_{v} \times \mathbb{R}_{t}\right)$ for some $\sigma \geq 0$, let $g \in L_{\text {loc }}^{q}\left(\mathbb{R}_{x}^{N} \times \mathbb{R}_{v} \times \mathbb{R}_{t}\right)$ if $q>1$, or let $g$ be a locally bounded measure on $\mathbb{R}_{x}^{N} \times \mathbb{R}_{v} \times \mathbb{R}_{t}$ if $q=1$. We assume that $f$ satisfies

$$
\frac{\partial f}{\partial t}+a(v) \cdot \nabla_{x} f=\frac{\partial g}{\partial v} \quad \text { in } \mathscr{D}^{\prime}\left(\mathbb{R}_{x}^{N} \times \mathbb{R}_{v} \times \mathbb{R}_{t}\right) .
$$

Then, let

$$
\theta=\frac{\alpha}{p^{\prime}}\left(\frac{\alpha}{p^{\prime}}+2-\frac{\alpha}{q^{\prime}}\right)^{-1}
$$

$r$ be given by

$$
\frac{1}{r}=\frac{\theta}{q}+\frac{1-\theta}{p}, \quad \tau=\theta+(1-\theta) \sigma .
$$

Then $\int f \psi d v \in W_{\mathrm{loc}}^{s, r}\left(\mathbb{R}_{x}^{N} \times \mathbb{R}_{t}\right)$ for all $s \in(\theta, \tau)$.

Remarks. (1) Similar results can be obtained if we replace (as in [13]) $\partial g / \partial v$ by

$$
\left(-\Delta_{x, t}+1\right)^{\nu / 2}\left(-\Delta_{v}+1\right)^{m / 2} g \text { with } \nu \in[0,1), m \geq 0 .
$$

(2) Of course, local bounds on $\int f \psi d v$ in $W^{s, r}$ depend on $s, p, q, \sigma, N$ and on local bounds on $f$ and $g$.

In order to simplify the presentation, we will not bother to keep track of the bounds in the proof of Theorem 4 and will only show that

$$
\rho \in W_{\text {loc }}^{s, p}\left(\mathbb{R}_{x}^{N} \times(0, \infty)\right) \cap C\left((0, \infty) ; W_{\text {loc }}^{s, 1}\left(\mathbb{R}^{N}\right)\right) .
$$


Further simplification will be achieved by observing that since $\rho^{0}$ and $\rho$ belong to $L^{\infty}$, by the finite speed of propagation of solutions $(1),(2)$, we may assume without loss of generality that $\rho^{0}$ and $\rho$ have bounded supports in $x$ (uniform in $t$ bounded in $[0, \infty)$ of $\rho$ ).

As we shall see, the proof of Theorem 4 uses Theorem A with $p=2, q=1$, some interpolation arguments together with a bootstrap argument made possible by the following property of entropy solutions of (1), (2).

Lemma 2. (i) Let $\psi \in L^{1} \cap L^{\infty}\left(\mathbb{R}^{N}\right), \psi \geq 0$. Then,

$$
\iint_{\mathbb{R}^{N} \times \mathbb{R}^{N}}|\rho(x, t)-\rho(y, t)| \psi(x-y) d x d y
$$

is a nonincreasing function of $t \geq 0$.

(ii) If $\rho^{0} \in W^{s, 1}\left(\mathbb{R}^{N}\right)$ for some $s \in(0,1)$, then $\|\rho(t)\|_{W^{s, 1}\left(\mathbb{R}_{x}^{N}\right)}$ is a nonincreasing function of $t \geq 0$.

Recall that

$$
\|\varphi\|_{W^{s, 1}\left(\mathbb{R}^{N}\right)}=\|\varphi\|_{L^{\mathrm{i}}\left(\mathbb{R}^{N}\right)}+\iint_{\mathbb{R}^{N} \times \mathbb{R}^{N}} \frac{|\varphi(x)-\varphi(y)|}{|x-y|^{N+s}} d x d y .
$$

Therefore, (ii) follows from (i) in a straightforward way. The proof of (i) is in fact a general (abstract) fact about $L^{1}$-contractive, translationinvariant semigroups. Indeed, we know from Kružkov's theory [21] that $\int_{\mathbb{R}^{N}}\left|\rho_{1}(t)-\rho_{2}(t)\right| d x$ is a nonincreasing function of $t \geq 0$ whenever $\rho_{1}, \rho_{2}$ are entropy solutions of (1), (2). In particular, since $\rho(\cdot-h, t)$ is the entropy solution of (1), (2) corresponding to $\rho^{0}(\cdot-h)$ for all $h \in \mathbb{R}^{N}$, we deduce that

$$
\int_{\mathbb{R}^{N}}|\rho(x, t)-\rho(x-h, t)| d x \text { is a nonincreasing function of } t>0 .
$$

And Lemma 2(i) follows upon multiplying by $\psi(h)$ and integrating with respect to $h \in \mathbb{R}^{N}$.

We offer a final observation before going into the computations that will yield Theorem 4: the kinetic formulation (5)-(7) is of course valid for $t>0$, while the "velocity-averaging" Theorem B requires $f$ and $g$ to be defined for $t \in \mathbb{R}$. This is easily resolved by applying Theorem B to $f \varphi(t), g \varphi(t)$ where $\varphi \in C^{\infty}(\mathbb{R}), \varphi \equiv 0$ for $t \leq 0$, and $\varphi \neq 1$ for $t \geq h>0$ where $h>0$ can be made arbitrarily small. In doing so, we add to the right-hand side of (39) a term of the form of $\varphi^{\prime}$ which does not affect the conclusion of Theorem B.

With all these simplifications and modifications in mind, we may proceed with the proof of Theorem 4; we use the kinetic formulation (5)-(7). Since $f \in L_{\text {loc }}^{2}\left(\mathbb{R}_{x}^{N} \times(0, \infty)\right)$, we may apply Theorem A with $q=1, p=2, \sigma=0$, and $\psi$ such that $\psi \equiv 1$ on $\left[-\left\|\rho^{0}\right\|_{L^{\infty}},\left\|\rho^{0}\right\|_{L^{\infty}}\right]$. Notice that the choice $q=1$, $p=2$ that we shall use repeatedly yields

$$
\theta=\frac{\alpha}{\alpha+4}, \quad r=\frac{\alpha+4}{\alpha+2}, \quad \tau=\frac{\alpha+4 \sigma}{\alpha+4} .
$$

We then deduce from Theorem A that $\rho \in W_{\text {loc }}^{s, r}\left(\mathbb{R}_{x}^{N} \times(0, \infty)\right)$ for all $s<\tau_{1}=$ $\alpha /(\alpha+4)$. 
In particular, $\rho \in W_{\text {loc }}^{s, 1}\left(\mathbb{R}_{x}^{N} \times(0, \infty)\right)$ for all $s<\tau_{1}$. By the compact support simplification and Lemma $2(\mathrm{ii})$, we deduce in fact that $\rho(t)$ is bounded in $W^{s, 1}\left(\mathbb{R}_{x}^{N}\right)$ for $t \geq \varepsilon(\forall \varepsilon>0)$. Then, since $\rho \in C\left([0, \infty) ; L^{1}\left(\mathbb{R}_{x}^{N}\right)\right)$, we deduce that $\rho \in C\left((0, \infty) ; W^{s, 1}\left(\mathbb{R}_{x}^{N}\right)\right)$ for all $s \in\left(0, \tau_{1}\right)$ and that $\rho \in$ $W^{s, 1}\left(\mathbb{R}_{x}^{N} \times(\varepsilon, 1 / \varepsilon)\right)$ (using equation (1) to recover the fractional regularity in $t)$ for all $s \in\left(0, \tau_{1}\right), \varepsilon \in(0,1)$. Since $f=\chi_{\rho}(v)$, we deduce easily that $f \in W^{s, 1}\left(\mathbb{R}_{x}^{N} \times \mathbb{R}_{v} \times(\varepsilon, 1 / \varepsilon)\right)$ for all $\varepsilon \in(0,1)$ and for all $s \in\left(0, \tau_{1}\right)$. For instance, one may observe that $f \in L^{1}\left(\mathbb{R}_{v} ; W^{s, 1}\left(\mathbb{R}_{x}^{N} \times(\varepsilon, 1 / \varepsilon)\right)\right)$, while $\partial f / \partial v$ is a bounded measure on $\mathbb{R}_{x}^{N} \times \mathbb{R}_{v} \times(0,1 / \varepsilon)$.

Next, using interpolation theory and the fact that $f \in L^{\infty}$, we deduce that $f \in W^{s, 2}\left(\mathbb{R}_{x}^{N} \times \mathbb{R}_{v} \times(\varepsilon, 1 / \varepsilon)\right)$ for all $s \in(0,1)$ and for all $s \in\left(0, \tau_{1 / 2}\right)$. At this stage, we may again apply Theorem A with $q=1, p=2$, and $\sigma=s$. We then deduce by the same argument as before that $\rho \in W_{\text {loc }}^{s, r}\left(\mathbb{R}_{x}^{N} \times(0, \infty)\right) \cap C((0, \infty)$; $\left.W^{s, 1}\left(\mathbb{R}_{x}^{N}\right)\right)$ for all $s \in\left(0, \tau_{2}\right)$ where $\tau_{2}=\left(\alpha+2 \tau_{1}\right) /(\alpha+4)$. Reiterating this argument, we conclude the proof of Theorem 4 since $\tau=\alpha /(\alpha+2)$ is the unique fixed point of the increasing map $\left(\tau_{1} \mapsto \tau_{2}\right)$.

\section{MOMENTS ESTIMATES AND BOUNDS ON ENTROPY SOLUTIONS}

In this section, we show how one can deduce from the kinetic formulation new $L_{\text {loc }}^{p}$ bounds on entropy solutions of multidimensional scalar conservation laws. If $N \geq 2$, such results seem to be new, while, if $N=1$, related results exist for convex flux $A$ (by the Lax-Oleinik formula) or for more general fluxes and we refer to Liu and Pierre [24], Dafermos [9], etc. In fact, if $N=1$, quite general results showing $L_{x}^{\infty}$ bounds on solutions for positive time $t$ can be deduced from the regularizing effect results shown in Lions [23]; indeed, one just has to "raise solutions at the Hamilton-Jacobi level" by considering $\int_{-\infty}^{x} \rho(y, t) d t$ and apply the results of [23]. However, all these one-dimensional results seem to be of a different nature than the ones that follow since we shall obtain results on $L^{p} \quad(|x| \leq R, t \in(0, R))$ bounds on solutions for all $R<\infty$. In fact, our results seem to be more related to dispersive local estimates for Schrödinger like equations as shown by Vega [34], Sjölin [30], Constantin, and Saut [8], etc. Our method of proof will be to adapt the method introduced by Perthame [27, 28], which provides local higher integrability in $v$ (or more precisely local in $x, t$, higher moments in $v$ ) for solutions of kinetic type equations by some kind of dispersion analysis.

We shall use the following condition on $a$ :

$$
|v|\left|a^{\prime}(v)\right| \leq\left\{\begin{array}{ll}
C|a(v)| & \text { if }|a(v)| \geq 1, \\
C|a(v)|^{1 / 2} & \text { if }|a(v)| \leq 1,
\end{array} \quad \text { on } \mathbb{R}, \text { for some } C \geq 0\right.
$$

Proposition 1. Under condition (40), the entropy solution $\rho$ of (1), (2), satisfies for all $r>0$ and for all $R \in(0, \infty)$

$$
\left.\int_{0}^{R} \int_{|x| \leq R}\left|\int_{0}^{\rho}\right| v\right|^{r}|a(v)|^{1 / 2} d v \mid d x d t \leq C\left\|\rho^{0}\right\|_{L^{1+r}\left(\mathbb{R}^{N}\right)}^{1+r}
$$


where $C \geq 0$ depends only on $A$ and $R$.

Remarks. (1) Since we have $\|\rho(t)\|_{L^{1+r}\left(\mathbb{R}^{N}\right)} \leq\left\|\rho^{0}\right\|_{L^{1+r}\left(\mathbb{R}^{N}\right)}$ for all $t \geq 0, r>0$, we immediately see that $\int_{0}^{\rho}|v|^{r} \mid a(v)^{1 / 2} d v$ can be replaced by any $\int_{0}^{\rho} \omega(v) d v$ where $\omega(v)=|v|^{r}|a(v)|$ when $|v|$ and $|a(v)|$ are large.

(2) In addition, the above remark shows that (41) yields some new information only when

$$
\left.\left.\left|\int_{0}^{t}\right| v\right|^{r}|a(v)|^{1 / 2} d v|| t\right|^{-(1+r)} \rightarrow+\infty \quad \text { as }|t| \rightarrow+\infty .
$$

In particular, if $|a(v)|$ "behaves like $|v|^{\alpha}$ " as $|v|$ goes to $+\infty$ for some $\alpha>0$, the above result yields a local bound in $L^{p}$ with $p=1+r+\alpha / 2$, representing thus a gain of $\alpha / 2$ in terms of the integrability exponents.

(3) The $L^{1}$ case and related questions Demangel and Serre [10]) seems to be out of the scope of the higher moment techniques.

Proof of Proposition 1. In order to simplify the presentation, we shall consider only the case when $r=1$ - the general case being handled in a similar manner. We then follow the method of proof introduced in Perthame [27, 28]. Let $\varphi, \psi$ denote two cut-off functions $\varphi, \psi: \varphi, \psi \in C_{0}^{\infty}\left(\mathbb{R}^{N}\right), 0 \leq \varphi, \psi \leq 1$ on $\mathbb{R}^{N}$, $\varphi=\psi=1$ if $|x| \leq R, \operatorname{Supp} \varphi, \operatorname{Supp} \psi \subset B_{2 R+1}$. We introduce a function $d(v)>0$ which is equal to $|a(v)|^{1 / 2}$ "far from" the set $\{a(v)=0\}$ such as, for instance, $d(v)=\max \left(\mid a(v)^{1 / 2}, 1\right)$. We now set $\Psi(x, v)=\psi(x / d(v))$ and deduce from (5)

$$
\frac{\partial}{\partial t}(f \Psi)+a(v) \cdot \nabla_{x}(f \Psi)=\frac{a(v)}{d(v)} \cdot \nabla_{x} \psi\left(\frac{x}{d(v)}\right) f+\Psi \frac{\partial m}{\partial v} .
$$

Since $f$ has the same sign as $v$, we can write the left-hand side of (41) as $\int_{0}^{R} \int_{|x| \leq R} \int_{\mathbb{R}_{v}} v|a(v)|^{1 / 2} f d v d x d t$. And writing equation (42) in integral form (along the characteristic lines $x-a(v) t$ ), we deduce

$$
\begin{aligned}
& \int_{\mathbb{R}} v|a(v)|^{1 / 2} \int_{0}^{R} \int_{\mathbb{R}^{N}} \varphi(x)(\Psi f)(x, v, t) d x d t d v \\
& =\int_{\mathbb{R}} v|a(v)|^{1 / 2} d v \int_{0}^{R} d t \int_{\mathbb{R}^{N}} d x \varphi(x) \\
& \quad \times\{(\Psi f)(x-a(v) t, v, 0) \\
& +\int_{0}^{t}\left[\frac{a(v)}{d(v)} \cdot\left(\nabla_{x} \psi f\right)(x-a(v) s, v, t-s)\right. \\
& \left.\left.\quad+\left(\Psi \frac{\partial m}{\partial v}\right)(x-a(v) s, v, t-s)\right] d s\right\} .
\end{aligned}
$$

Noticing that

$$
\left(\frac{\partial m}{\partial v}\right)(x-a(v) s, v, t-s)=\left(\frac{\partial}{\partial v}+s a^{\prime}(v) \cdot \nabla_{x}\right) \cdot(m(x-a(v) s, v, t-s)) \text {, }
$$


the last term of the right-hand side of (43) may be written after an integration by parts as

(44)

$$
\begin{array}{rl}
-\int_{0}^{R} d & t \int_{\mathbb{R}^{N}} d x \varphi(x) \int_{0}^{t} d s \int_{\mathbb{R}} d v m(x-a(v) s, v, t-s) \\
\times & {\left[\frac{d}{d v}\left(v\left(|a(v)|^{1 / 2}\right) \Psi+\left(|v||a(v)|^{1 / 2}\right) \nabla \Psi \cdot \frac{\partial}{\partial v}\left(\frac{x-a(v) s}{d(v)}\right)\right]\right.} \\
& -\int_{\mathbb{R}} v|a(v)|^{1 / 2} d v \int_{\mathbb{R}^{N}} d x \int_{0}^{R} d t \int_{0}^{t} d s s a^{\prime}(v) \cdot\left(\frac{\varphi(x)}{d(v)} \nabla \Psi+\nabla \varphi(x) \Psi\right) .
\end{array}
$$

All these terms and the two first terms of the right-hand side of (43) can be treated in a similar way, and thus we will present the analysis of only the relevant ones. In this treatment, we shall use the following change of variables. For a fixed $v$ with $|a(v)| \geq 1$, we set

$$
u=|a(v)| s, \quad x=x^{\prime}+y e, \quad y \in \mathbb{R}, \quad e=a(v)|a(v)|^{-1} .
$$

The first term we treat is the first term of the right-hand side of (43). Then, before performing the integration with respect to $v$, we find for $|a(v)| \geq 1$

$$
\begin{aligned}
& v|a(v)|^{1 / 2} \int_{0}^{R} \int_{\mathbb{R}^{N}} \varphi \Psi f(x-a(v) s, v, 0) d x d s \\
& \leq v|a(v)|^{1 / 2} \int_{\mathbb{R}^{N} \times \mathbb{R}^{N-1}} \varphi\left(x^{\prime}+y e\right) \\
& \times \int_{\mathbb{R}} f\left(x^{\prime}+e(y-u), v, 0\right) \frac{d u}{|a(v)|} d y d x^{\prime} \\
& \leq \int_{\mathbb{R}^{n-1} \times \mathbb{R}} v f\left(x^{\prime}+z e, v, 0\right) d x d z \int_{\mathbb{R}} \varphi\left(x^{\prime}+y e\right) d y \\
& \leq C(R) \int_{\mathbb{R}^{N}} v f^{0}(x, v) d x .
\end{aligned}
$$

For $|a(v)| \leq 1$, the same estimate holds with a straightforward verification that we skip. Performing the last integration in $v$, we find the desired estimate since $\int_{\mathbb{R}_{x}^{N}} \int_{\mathbb{R}_{v}} v f^{0}(x, v) d x d v=\left\|\rho^{0}\right\|_{L^{2}\left(\mathbb{R}^{N}\right)}^{2}$.

The second term we treat is the second term of the right-hand side of (43). Again, before the integration with respect to $v$, this term is bounded by

$$
\begin{gathered}
C v|a(v)|^{3 / 2} d(v)^{-1} \int_{0}^{R} \int_{\mathbb{R}^{N}} \int_{0}^{R} \varphi(x) f(x-a(v) s, v, \tau) d s d x d \tau \\
\leq C(R)|a(v)|^{1 / 2} / d(v) \int_{0}^{R} \int_{\mathbb{R}^{N}} v f(x, v, \tau) d x d \tau
\end{gathered}
$$

using the same argument as above. This last quantity after integrating with respect to $v$ yields $\int_{0}^{R} \int_{\mathbb{R}^{N}}|\rho(x, \tau)|^{2} d x d \tau$, which is bounded by $R\left\|\rho^{0}\right\|_{L^{2}\left(\mathbb{R}^{N}\right)}^{2}$. 
The last term we consider is the last term in (44)-the others being treated similarly. For $v$ fixed, we obtain

$$
\begin{aligned}
& \mid v\left(\left.a(v)\right|^{1 / 2} a^{\prime}(v) \cdot \int_{\mathbb{R}^{N}} d x\right. \\
& \times \int_{0}^{R} d t \int_{0}^{t} \nabla \varphi(x) \psi\left(\frac{x-a(v) s}{d(v)}\right) m(x-a(v) s, v, t-s) s d s \mid \\
& \quad \leq 2(R+1)|v|\left|a^{\prime}(v)\right||a(v)|^{-1 / 2} d(v) \\
& \quad \times \int_{\mathbb{R}^{N}} \int_{0}^{R} \int_{0}^{R} m(x-a(v) s, v, \tau) d \tau d s d x,
\end{aligned}
$$

since we deduce from the restrictions on the supports of $\varphi$ and $\psi$

$$
|x| \leq 2 R+1, \quad|a(v)| s \leq|x|+d(v) \leq 2 R+1+d(v) \leq 2(R+1) d(v) .
$$

We may now perform the same analysis as above on the right-hand side of (47) and find when $|a(v)| \geq 1$ an upperbound of the form

$$
C(R) \int_{\mathbb{R}^{N}} \int_{0}^{R} m(x, v, \tau) d x d \tau
$$

while, if $|a(v)| \leq 1$, we also obtain such an upperbound, using in each case the condition (40). Then, integrating this bound with respect to $v$ and using the first bound in (13), we conclude easily.

\section{RElated EQuations}

In this section, we briefly mention how to adapt the kinetic formulation (5)(7) to related classes of equations. We will present here two classes of problems that we know how to treat, namely, multidimensional scalar conservation laws with source terms or degenerate second-order scalar conservation laws. Before going into a more precise description, we want to emphasize the fact that we do not know if or how one can adapt our approach to systems of conservation laws except for very special ones like the one-dimensional monoatomic isentropic gas dynamics system for which we presented some results in our announcement (Comptes-Rendus Acad. Sci. Paris Sér. I 312 (1991), 9-102).

Next, let us begin with multidimensional scalar conservation laws with source terms, namely,

$$
\frac{\partial \rho}{\partial t}+\sum_{i=1}^{N} \frac{\partial}{\partial x_{i}}\left(A_{i}(\rho)\right)=A(\rho) \text { for } t \geq 0, x \in \mathbb{R}^{N}
$$

with initial condition (4). Here, the fluxes $A_{i}$ satisfy the same conditions as in the introduction, while $A \in C^{1}(\mathbb{R} ; \mathbb{R}$ ) with $A(0)=0$ (for example). Entropy conditions then become

$$
\frac{\partial}{\partial t} S(\rho)+\sum_{i=1}^{N} \frac{\partial}{\partial x_{i}}\left(\eta_{i}(\rho)\right) \leq A(\rho) S^{\prime}(\rho) \text { in } \mathscr{D}^{\prime}
$$

for all convex functions $S$, where $\eta_{i}$ is still given by (3). 
In order to avoid $L^{\infty}$ blow-up difficulties, we assume for simplicity

$$
(\operatorname{sign} t) A(t) \leq C_{0} \text { on } \mathbb{R} \text {, for some } C_{0} \geq 0 \text {, }
$$

or

$$
\exists \alpha \leq 0 \leq \beta ; \quad \alpha \leq \rho^{0}(x) \leq \beta \quad \text { in } \mathbb{R}^{N}, \quad A(\alpha) \geq 0 \geq A(\beta) .
$$

In both cases, there exists a unique solution

$$
\rho \in L^{\infty}\left(\mathbb{R}_{x}^{N} \times(0, T)\right) \cap C\left([0, \infty) ; L^{1}\left(\mathbb{R}_{x}^{N}\right)\right)
$$

$(\forall T<\infty)$ of $(48),(49),(4)$.

Then, the kinetic formulation $(5)-(7)$ is to be modified as follows: we still impose (6) and (7) and we replace the kinetic equation (5) by

$$
\frac{\partial f}{\partial t}+a(v) \cdot \nabla_{x} f+A(v) \frac{\partial f}{\partial v}=\frac{\partial m}{\partial v} \text { in } \mathscr{D}^{\prime} .
$$

Then, it is possible to adapt all our arguments and methods to recover the exact analogues of all the results above.

Another extension (which can be combined of course with the preceding one by adding a source term on the right-hand side) concerns second-order, possibly degenerate, parabolic equations in conservation form

$$
\frac{\partial \rho}{\partial t}+\sum_{i=1}^{N} \frac{\partial}{\partial x_{i}}\left(A_{i}(\rho)\right)-\sum_{i, j=1}^{N} \frac{\partial^{2}}{\partial x_{i} \partial x_{j}}\left(A_{i j}(\rho)\right)=0 \text { in } \mathbb{R}_{x}^{N} \times(0, \infty)
$$

together with initial condition (4). Here and below, we assume that $A_{i}, A_{i j} \in$ $C_{\text {loc }}^{1,1}(\mathbb{R})$ and that $\left(A_{i j}\right)$ satisfies the degenerate ellipticity condition

$$
\left(A_{i j}^{\prime}(t)\right) \geq 0 \text { in } \mathbb{R},
$$

i.e.,

$$
\sum_{i, j=1}^{N} A_{i j}^{\prime}(t) \xi_{i} \xi_{j} \geq 0 \quad \text { for all } t \in \mathbb{R}, \xi \in \mathbb{R}^{N} .
$$

Of course, the above class of equations contains as special cases the scalar conservation laws (1) but also the heat equation (take $A_{i} \equiv 0, A_{i j} \equiv \delta_{i j}$ ) or various porous media type equations (take $A_{i} \equiv 0, A_{i j}(t) \equiv \varphi(t) \delta_{i j}$ with $\varphi$ nondecreasing). There does not seem to be a general existence, uniqueness, and stability theory for such a general class of equations. Part of the difficulties is the very notion of solutions (say in $L^{\infty}\left(\mathbb{R}_{x}^{N} \times(0, \infty)\right) \cap C\left([0, \infty) ; L^{1}\left(\mathbb{R}_{x}^{N}\right)\right.$ ): indeed, if $A_{i} \equiv 0, A_{i j}(t) \equiv \varphi(t) \delta_{i j}$, weak solutions, i.e., solutions of $(53)$ in distributions sense, are known to exist and to be unique $(.$.$) , and we refer to$ Brézis and Crandall [6], Bènilan and Crandall [3, 4] (and the references therein) for rather general uniqueness and stability results in that direction. On the other hand, if $A_{i j} \equiv 0$, entropy conditions are necessary for uniqueness! In general, the analogue of entropy conditions exists here, namely,

$$
\frac{\partial S(\rho)}{\partial t}+\sum_{i=1}^{N} \frac{\partial}{\partial x_{i}}\left(\eta_{i}(\rho)\right)-\sum_{i, j=1}^{N} \frac{\partial^{2}}{\partial x_{i} \partial x_{j}}\left(\eta_{i j}(\rho)\right) \leq 0 \text { in } \mathscr{D}^{\prime}
$$


where $\eta_{i}(t)=\int_{0}^{t} S^{\prime}(s) A_{i}^{\prime}(s) d s, \eta_{i j}(t)=\int_{0}^{t} S^{\prime}(s) A_{i j}^{\prime}(s) d s$, for all convex functions $S$.

It is not difficult to build one solution of (53), (55), (4) in $L^{\infty}\left(\mathbb{R}_{x}^{N} \times(0, \infty)\right) \cap$ $C\left([0, \infty) ; L^{1}\left(\mathbb{R}_{x}^{N}\right)\right)$ or even an $L^{1}$-contraction, translation invariant, semigroup solution of (53), (55). In particular, it is possible to build a solution that satisfies in addition that, if $\rho^{0}$ has finite total variation, then $\rho(t)$ for all $t \geq 0$ has finite total variation and its total variation is a nonincreasing function of $t \geq 0$. However, the main open question seems to be the uniqueness of such solutions (or semigroups). We do not know how to solve this problem, but we hope that the kinetic formulation of (53), (55) we are going to present might turn out to be useful in that respect. Anyway, we will also indicate some applications of that formulation to surprisingly general compactness results.

The kinetic formulation is in fact very close to (5)-(7) and is given by (6), (7) and

$$
\begin{aligned}
\frac{\partial f}{\partial t}+\sum_{i=1}^{N} a_{i}(v) \cdot \frac{\partial f}{\partial x_{i}}-\sum_{i, j=1}^{N} a_{i j}(v) \frac{\partial^{2} f}{\partial x_{i} \partial x_{j}}=\frac{\partial m}{\partial v} \\
\quad \text { in } \mathscr{D}^{\prime}\left(\mathbb{R}_{x}^{N} \times \mathbb{R}_{v} \times(0, \infty)\right)
\end{aligned}
$$

where $a_{i}(t)=A_{i}^{\prime}(t), a_{i j}(t)=A_{i j}^{\prime}(t)$ on $\mathbb{R}$. Of course, the corresponding initial condition is (9).

It is worth remarking that the above formulation applies to heat or porous medium equation and that in the example of the heat equation $\left(A_{i} \equiv 0, A_{i j} \equiv\right.$ $\left.\delta_{i j}\right)$ the measure $m$ is given by $\left|\nabla_{x} \rho(x, t)\right|^{2} \cdot \delta_{0}(v-\rho(x, t))$. Moreover, any solution of (53) satisfies (56) with

$$
m=\delta_{0}(v-\rho(x, t)) a_{i j}(v) \frac{\partial \rho}{\partial x_{i}} \frac{\partial \rho}{\partial x_{j}}
$$

on open subsets of $\mathbb{R}_{t}^{+} \times \mathbb{R}_{x}^{N}$ where it is lipschitz continuous.

Next, the proof of Theorem 1 can be adapted to the above general situation proving thus that (53), (55) is equivalent to (56), (6), and (7). Uniqueness of solutions, however, is a general open problem even if some partial results can probably be deduced from Volpert and Hudjaev [35].

Let us conclude by mentioning an application to $L_{\mathrm{loc}}^{1}$ compactness of bounded solutions of (53), (55) which holds under the nondegeneracy condition

$$
\begin{aligned}
& \text { meas }\left\{v \in \mathbb{R} \mid \tau+\sum_{i} a_{i}(v) \xi_{i}=0, \sum_{i, j} a_{i j}(v) \xi_{i} \xi_{j}=0\right\}=0 \\
& \text { for all }(\tau, \xi) \in \mathbb{R} \times \mathbb{R}^{N} \text { with } \tau^{2}+|\xi|^{2}=1 .
\end{aligned}
$$

This follows directly from a velocity-averaging compactness result presented in the Appendix (Theorem C) which is a variant of the results of [13]. Additional regularity can also be obtained, but we will not pursue this here. Let us finally mention that if we consider sequences of solutions $\rho^{\varepsilon}$ corresponding to fluxes $\left(A_{i}^{\varepsilon}, A_{i j}^{\varepsilon}\right)$ converging to $\left(A_{i}, A_{i j}\right)$ as $\varepsilon$ going to 0 (assuming $(54)$ for all $\varepsilon>0$ ), 
$\rho^{\varepsilon}$ is compact in $L_{\text {loc }}^{1}$ as soon as the following condition holds for all $R$

$$
\begin{array}{r}
\text { meas }\left\{v /|v| \leq R,\left|\tau+\sum_{i} a_{i}^{\varepsilon}(v) \xi_{i}\right| \leq \delta, \sum_{i, j} a_{i j}^{\varepsilon}(v) \xi_{i} \xi_{j} \leq \delta\right\} \rightarrow 0 \\
\text { as } \delta \rightarrow 0_{+}, \text {uniformly in } \varepsilon .
\end{array}
$$

\section{APPENDIX. VELOCITY-AVERAGING REVISITED}

We briefly explain how the proofs of Theorems A and B are adapted from those made in [13]. First of all, Theorem $A$ is proven exactly as in [13], the only modification being the estimate

$$
\int_{-R}^{+R} \frac{1}{|\tau+a(v) \cdot \xi|^{m}} 1_{|\tau+a(v) \cdot \xi| \geq \delta} d v \leq C \delta^{\alpha-m}
$$

for all $R \in(0, \infty), m>1, \delta \in(0,1)$, for some constant $C>0$ depending only on $R, m$, and $x$. This is easily shown by introducing: $\mu(t)=$ meas $\{v \in(-R, R),|\tau+a(v) \cdot \xi| \leq t\}$ for $t \geq 0$. Then, the above integral may be written as

$$
\begin{aligned}
\int_{\delta}^{\infty} \frac{d \mu(t)}{t} & =-\frac{\mu(\delta)}{\delta^{m}}+\int_{\delta}^{\infty} \frac{\mu(t)}{t^{1+m}} d t \leq \int_{\delta}^{\infty} \frac{\mu(t)}{t^{1+m}} d t \\
& \leq-C \int_{\delta}^{\infty} \frac{t^{\alpha}}{t^{1+m}} d t \leq C \delta^{\alpha-m} .
\end{aligned}
$$

We now briefly indicate how Theorem B is deduced from the arguments of [13]. Let $\zeta \in C_{0}^{\infty}(\mathbb{R})$ with $\zeta \equiv 1$ near $0, \operatorname{Supp} \zeta \subset(-1,+1)$. Let $\rho_{\delta}=\frac{1}{\delta} \rho(\dot{\bar{\delta}})$ with $0 \leq \rho \in C_{0}^{\infty}(\mathbb{R}), \int_{\mathbb{R}} \rho d x=1$, and set $\psi_{\delta}=\psi * \rho_{\delta}$. Observe, of course, that $\operatorname{Supp} \psi_{\delta} \subset \operatorname{Supp} \psi+\operatorname{Supp} \rho_{\delta}$ so that (35) yields

$$
\operatorname{meas}\left\{v \in \operatorname{Supp} \psi_{\delta},|\tau+a(v) \cdot \xi| \leq \delta\right\} \rightarrow 0 \quad \text { as } \delta \rightarrow 0_{+}
$$

for all $(\tau, \xi) \in \mathbb{R}^{0} \times \mathbb{R}^{N}$ with $\tau^{2}+|\xi|^{2}=1$. Then, we deduce

$$
\begin{aligned}
& \sup \left\{\operatorname{meas}\left\{v \in \operatorname{Supp} \psi_{\delta},|\tau+a(v) \cdot \xi| \leq \delta\right\} /\right. \\
& \left.(\tau, \xi) \in \mathbb{R} \times \mathbb{R}^{N}, \tau^{2}+|\xi|^{2}=1\right\}=0 .
\end{aligned}
$$

At this stage, denoting by $\mathscr{F}$ and $\mathscr{F}^{-1}$ the Fourier and the inverse Fourier transforms in $(x, t)$, we may write, after having localized (34) so that $f, g$ have compact supports in $(x, t)$,

$$
\begin{aligned}
\int_{\mathbb{R}} f \psi d v= & \int_{\mathbb{R}} f\left(\psi-\psi_{\delta}\right) d v+\int_{\mathbb{R}} \psi_{\delta^{\mathscr{F}}}\left(\zeta\left(\frac{\tau+a(v) \cdot \xi}{\delta}\right) \mathscr{F} f\right) d v \\
& +\int_{\mathbb{R}} \psi_{\delta} \mathscr{F}^{-1}\left(1-\zeta\left(\frac{\tau+a(v) \cdot \xi}{\delta}\right) \mathscr{F} f\right) d v
\end{aligned}
$$

The last term can be shown to be compact in $L^{p}$ because of (34) as in [13]. Again, as in [13], the second term can be shown to be small in $L^{p}$ (uniformly 
in $f$ ), as $\delta$ goes to 0 because of the above condition. Finally, since $\Psi_{\delta} \rightarrow \psi$ in $L^{p^{\prime}}(\mathbb{R})$, we deduce easily

$$
\begin{aligned}
\left\|\int_{\mathbb{R}} f\left(\psi-\psi_{\delta}\right) d v\right\|_{L^{p}} & \leq\left\|\psi-\psi_{\delta}\right\|_{L^{p^{\prime}}(\mathbb{R})}\left\|\left(\int_{\mathbb{R}}|f|^{p} d v\right)^{1 / p}\right\|_{L^{p}} \\
& \leq C\left\|\psi-\psi_{\delta}\right\|_{L^{p^{\prime}}(\mathbb{R})}
\end{aligned}
$$

for some $C \geq 0$ independent of $f$. This yields the conclusion of Theorem $\mathrm{B}$.

Finally, we present a result (proven exactly as in [13] with the above modifications) concerning equations of the form

$$
\begin{aligned}
\frac{\partial f}{\partial t} & +\sum_{i=1}^{N} a_{i}(v) \frac{\partial f}{\partial x_{i}}-\sum_{i, j=1}^{N} a_{i j}(v) \frac{\partial^{2} f}{\partial x_{i} \partial x_{j}} \\
& =\left(-\Delta_{x, t}+1\right)^{1 / 2}\left(-\Delta_{v}+1\right)^{r / 2} g \text { in } \mathscr{D}^{\prime}
\end{aligned}
$$

where $r \geq 0, g$ belongs to a compact set of $L^{p}\left(\mathbb{R}_{x}^{N} \times \mathbb{R}_{v} \times \mathbb{R}_{t}\right), f$ is bounded in $L_{\text {loc }}^{p}\left(\mathbb{R}_{x}^{N} \times \mathbb{R}_{v} \times \mathbb{R}_{+}\right)$with $1<p \leq 2$ and $a_{i}, a_{i j}$ are smooth. Let $\psi \in L^{p^{\prime}}\left(\mathbb{R}_{v}\right)$ with compact support.

Theorem C. With the above notation and assumptions and if in addition we have

$$
\begin{aligned}
& \operatorname{meas}\left\{v \in \operatorname{Supp} \psi, \tau+a(v) \cdot \xi=0, \sum_{i, j=1}^{N} a_{i j}(v) \xi_{i} \xi_{j}=0\right\} \\
& \qquad \text { for all }(\tau, \xi) \in \mathbb{R} \times \mathbb{R}^{N} \text { with } \tau^{2}+|\xi|^{2}=1,
\end{aligned}
$$

then, $\int_{\mathbb{R}} f \psi d v$ belongs to a compact set of $L_{\mathrm{loc}}^{p}\left(\mathbb{R}_{x}^{N} \times \mathbb{R}_{t}\right)$.

\section{REFERENCES}

1. C. Bardos, F. Golse, and D. Levermore, Fluid dynamic limits of kinetic equations. II:, Convergence proofs for the Boltzmann equation. (preprint)

2. C. Bardos, F. Golse, B. Perthame, and R. Sentis, The non-accretive radiative transfer equations: existence of solutions and Rosseland approximation, J. Funct. Anal. 77 (1988), 434-460.

3. Ph. Bènilan and M. G. Crandall, Regularizing effects of homogeneous evolution equations, Contributions to Analysis and Geometry, John Hopkins Univ. Press, Baltimore, MD, 1981, pp. 23-39.

4. $\ldots$, The continuous dependence on $\varphi$ of solutions of $u_{t}-\Delta \varphi(u)=0$, Indiana Univ. Math. J. 30 (1981), 162-177.

5. Y. Brenier, Résolution d'équations d'évolution quasilinéaires en dimension $N$ d'espace à l'aide d'équations linéaires en dimension $N+1$, J. Differential Equations 50 (1983), 375-390.

6. H. Brézis and M. G. Crandall, Uniqueness of solution of the initial-value problem for $u_{t}-$ $\Delta \varphi(u)=0$, J. Math. Pures Appl. (9) 58 (1979), 153-163.

7. C. Cercignani, The Boltzmann equation and its applications, Springer-Verlag, New York, Berlin and Heidelberg, 1988.

8. P. Constantin and J. C. Saut, Local smoothing properties of Schrödinger equations, Indiana Univ. Math. J. 38 (1989), 791-810. 
9. C. Dafermos, Regularity and large time behaviour of solutions of a conservation law without convexity, Proc. Roy. Soc. Edinburgh Sect. A 99 (1985), 201-239.

10. F. Demangel and D. Serre, Non-vanishing singular parts of hyperbolic equations, prépublications 90-18, Univ. Paris-Sud and C. R. Acad. Sci. Paris Série I 309 (1989), no. 19, 975-978.

11. R. J. DiPerna, Measure-valued solutions to conservation laws, Arch. Rational Mech. Anal. 88 (1985), 223-270.

12. R. J. DiPerna and P. L. Lions, Global weak solutions of Vlasov-Maxwell systems, Comm. Pure Appl. Math. 42 (1989), 729-757.

14. B. Engquist, Personal communication.

15. P. Gérard, Moyenne de solutions d'équations aux dérivées partielles, Séminaire EDP 19861987, École Polytechnique, Palaiseau, 1988.

16. P. Gérard, Moyennisation et régularité deux-microlocale, Ann. Sci. Ecole Norm. Sup. (4) 23 (1990), no. 7, 89-121.

17. Y. Giga and T. Miyakawa, A kinetic construction of global solutions of first-order quasilinear equations, Duke Math. J. 50 (1983), 505-515.

18. F. Golse, P. L. Lions, B. Perthame, and R. Sentis, Regularity of the moments of the solution of a transport equation, J. Funct. Anal. 76 (1988), 110-125.

19. F. Golse, B. Perthame, and R. Sentis, Un résultat de compacité pour les équations du transport, C. R. Acad. Sci. Paris, Sér. I Math. 301 (1985), 341-344.

20. F. Golse and F. Poupaud, Limite fluide de l'équation de Boltzmann des semi-conducteurs pour une statistique de Fermi-Dirac. (to appear in Comm. Pure Appl. Math.)

21. S. N. Kružkov, First-order quasilinear equations in several independent variables, Math. USSR-Sb. 10 (1970), 217-243.

22. P. D. Lax, Hyperbolic systems of conservation laws. II, Comm. Pure Appl. Math. 10 (1957), 537-566.

23. P. L. Lions, Regularizing effects for first-order Hamilton-Jacobi equations, Appl. Anal. 20 (1985), 283-308.

24. T. P. Liu and M. Pierre, Source-solutions and asymptotic behavior in conservation laws, J. Differential Equations 51 (1984), 419-441.

25. F. Murat, Compacité par compensation, Ann. Scuola Norm. Sup. Pisa Cl. Sci. (4) 5 (1978), 489-507.

26. O. Oleinik, The Cauchy problem for nonlinear equations in a class of discontinuous functions, Dokl. Akad. Nauk SSSR 95 (1954), 451-454; English transl. Amer. Math. Soc. Transl. Ser. 2, vol. 42, Amer. Math. Soc., Providence, RI, 1964, pp. 7-12.

27. B. Perthame, Global existence of solutions to the BGK model of Boltzmann equations, J. Differential Equations 81 (1989), 191-205.

28. __ Higher moments for kinetic equations; Applications to Vlasov-Poisson and FokkerPlanck equations, Math. Methods Appl. Sci. 13 (1990), 441-452.

29. B. Perthame and E. Tadmor, A kinetic equation with kinetic entropy functions for scalar conservation laws, Comm. Math. Phys. 136 (1991), 501-517.

30. P. Sjölin, Regularity of solutions to Schrödinger equations, Duke Math. J. 55 (1987), 699715.

31. J. Smoller, Shock waves and reaction-diffusion equations, Springer, Berlin, 1982.

32. L. Tartar, Une nouvelle méthode de résolution d'équations aux dérivées partielles nonlinéaires, Lecture Notes in Math., vol. 665, Springer, Berlin, 1977, pp. 228-241.

33. Compensated compactness and applications to partial differential equations, Nonlinear analysis and mechanics, Heriot-Watt Sympos., Vol. 4 (R. J. Knopps, ed.), Research Notes in Math., Vol. 39, Pitman, Boston and London, 1975, pp. 136-211.

34. L. Vega, Schrödinger equations: Pointwise convergence to the initial data, Proc. Amer. Math. Soc. 102 (1988), 874-878. 
35. A. I. Volpert and S. I. Hudjaev, Analysis in classes of discontinuous functions, and the equations of mathematical physics, Izdat. "Nauka", Moscow, 1975, p 394. (Russian)

36. K. Zumbrun, Asymptotic behavior for systems of nonconvex conservation laws, Ph.D. Thesis, Courant Institute, New York University.

37. P. L. Lions, B. Perthame, and E. Tadmor, Kinetic formulation of the isentropic gas dynamics and p-systems. (preprint)

ABSTRACT. We present a new formulation of multidimensional scalar conservation laws, which includes both the equation and the entropy criterion. This formulation is a kinetic one involving an additional variable called velocity by analogy. We also give some applications of this formulation to new compactness and regularity results for entropy solutions based upon the velocity-averaging lemmas. Finally, we show that this kinetic formulation is in fact valid and meaningful for more general classes of equations like equations involving nonlinear second-order terms.

Ceremade, Université Paris-Dauphine, Place de Lattre de Tassigny, 75775 Paris Cedex 16, FRANCE

Département de Mathématiques, Université d'Orléans, B. P. 6759, 45067 Orléans Cedex 2, France

School of Mathematical Sciences, Tel Aviv University, Tel Aviv 69978, IsRä̈l 